\title{
High-Throughput Automated Injection of Individual Biological Cells
}

\author{
Wenhui H. Wang†, Member, IEEE, Xinyu Y. Liu†, Student Member, IEEE, and Yu Sun, Senior Member, IEEE
}

\begin{abstract}
The ability of efficiently delivering soluable/insoluable drug compounds or biomolecules into individual biological cells and quantifying their cellular responses is important for genetics, proteomics, and drug discovery. This paper presents a fully automated system for zebrafish embryo injection, which overcomes the problems inherent in manual injection, such as human fatigue and large variations in success rates due to poor reproducibility. Based on "looking-then-moving" control, the microrobotic system performs injection at a speed of 15 zebrafish embryos (chorion unremoved) per minute. Besides a high injection speed that compares favorably with that of a highly proficient injection technician, a vacuum-based embryo holding device enables fast immobilization of a large number of zebrafish embryos, shortening the embryo patterning process from minutes to seconds. The recognition of embryo structures from image processing identifies a desired destination inside the embryo for material deposition, together with precise motion control resulting in a success rate of $100 \%$. Carefully tuning suction pressure levels as well as injection and retraction speeds produced a high survival rate of $98 \%$. The quantitative performance evaluation of the automated system was based on the continuous injection of $\mathbf{2 5 0}$ zebrafish embryos. The technologies can be extended to other biological injection applications such as the injection of mouse embryos, Drosophila embryos, and C. elegans to enable high-throughput biological and pharmaceutical research.
\end{abstract}

Note to Practitioners-The recent growth in the number of laboratories and companies using zebrafish in vertebrate developmental genetics and pharmaceutical research has been exponential. The injection of thousands of zebrafish embryos is required on a daily basis in a moderate-sized zebrafish laboratory/company, for applications such as embryonic development studies, mutation screening to identify genes, and drug molecule screening for drug discovery. The fully automated injection system and the embryo holding devices presented in this paper were developed to meet the requirements of high throughput and high reproducibility. Experimental results demonstrate that the system is reliable, operates at a high speed, and provides a high success rate and survival rate, capable of replacing or assisting a human operator for large-scale cell injection.

Index Terms-Cell injection, computer vision, drug delivery, genetics, high-throughput, microrobotic control, molecule screening, success rate, survival rate, zebrafish embryos.

Manuscript received January 01, 2007; revised May 28, 2007. First published March 14, 2008; current version published April 01, 2009. This paper was recommended for publication by Associate Editor M. Zhang and Editor D. Meldrum upon evaluation of the reviewers' comments. This work was supported by the Natural Sciences and Engineering Research Council of Canada and by the Ontario Ministry of Research and Innovation.

The authors are with the Advanced Micro and Nanosystems Laboratory, University of Toronto, ON M5S 3G8, Canada (e-mail: sun@mie.utoronto.ca)

Color versions of one or more of the figures in this paper are available online at http://ieeexplore.ieee.org.

Digital Object Identifier 10.1109/TASE.2008.917136

$†$ Shared first authorship.

\section{INTRODUCTION}

$\mathbf{T}$ HE PAST decades have witnessed the booming biological research on drug discovery for treating diseases such as cancers and heart diseases. Since many diseases originate from single pathological cells, many efforts have been spent on drug discovery for controlling and curing these pathological cells [1]-[4]. For investigating specific cellular responses, drug compounds and biomolecules need to be delivered into individual biological cells in a precise and dose-controllable manner. For instance, nucleic acid based molecules such as short interfering RNA (siRNA) and antisense oligonucleotides (AS-ON), which inhibit cancer-related gene expression, need to be delivered into cancer cells to evaluate their effectiveness [5].

Many technologies have been developed for cellular drug delivery, including ultrasound technique [6], electroporation [7], nanovector-based delivery [8], [9], and mechanical microinjection [10]. Among these techniques, microinjection is effective in delivering macromolecules of a soluble or insoluble compound into almost any cell type with high rates of cell viability [11]. Importantly, when automated, microinjection would be capable of precisely, reproducibly depositing drug molecules at a specific site inside cells, permitting cellular-function-targeted drugs to directly affect cell development and their functions observed.

In order to test cellular responses to a compound for molecule screening, the injection of thousands of cells is required within a short time period (e.g., within $1.5 \mathrm{~h}$ after fertilization, before the 16-cell stage for zebrafish embryo injection). The laborious task of current manual injection easily causes fatigue in injection technicians and hinders performance consistency and success rates [12]-[16], calling for fully automated, high-throughput injection systems.

Many attempts have been made to leverage robotic technologies to facilitate the process of cell injection. Microrobot-assisted (i.e., teleoperated) cell injection systems have been developed, where microrobots/micromanipulators are controlled by an operator to conduct "human-in-loop" cell injection [17]-[21]. Although the microrobots can to a certain extent facilitate cell injection by a human operator without long training, human involvement still exists in the process of cell injection, resulting in a low throughput and reproducibility.

A visually servoed microrobotic mouse embryo injection system was demonstrated, using a holding micropipette for immobilizing a single mouse embryo, and a visually servoed microrobot for automated cell injection [10]. However, switching from one embryo to another was conducted manually. A semi-automated high-throughput Drosophila embryo injection system was reported recently, where a single surface micromachined microelectromechanical systems (MEMS) needle was used as an injector [22]. One drawback of this system is that manual alignment of the injector and glass slide results in alignment errors that would greatly influence the injection performance. The low stiffness of the MEMS injector 


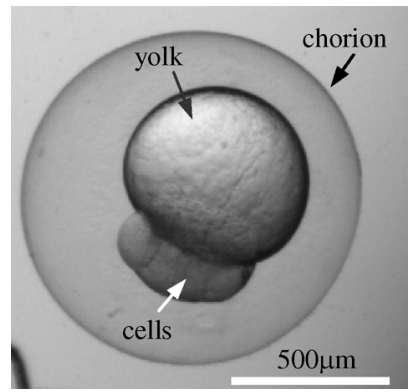

Fig. 1. Structure of a zebrafish embryo.

requires that the chorion (i.e., the outer membrane) must be removed before injection is conducted. Additionally, randomly dispersing embryos slows down the injection speed due to embryo searching.

Among many biological models, the zebrafish has emerged as an important model organism for development and genetic studies as well as drug discovery. The zebrafish has the advantages of similarities in the major organs to humans, external fertilization and development, short development period, and the transparency of embryos (Fig. 1), making it easy to observe the fate of individual cells during development [23]. The objective of this research is to develop a fully automated zebrafish embryo injection system to enable high-throughput genetic research and drug molecule screening.

Targeting high-throughput cell injection, MEMS-based microneedle arrays have been developed to perform parallel cell injection [24]-[28]. Although the concept of using microneedle arrays for parallel cell injection is appealing, several hurdles prevent their practical application. First, precisely aligning microneedles with regularly positioned cells is difficult. Manual alignment (in-plane or $X-Y$ alignment) through microscopic observation from an off-optical-axis angle does not guarantee a high accuracy. Second, determining the vertical distance (out-of-plane or $Z$ ) between microneedle tips and cells is difficult. Size differences from one cell to another (e.g., zebrafish embryos can differ by $200-300 \mu \mathrm{m}$ ) make vertical alignment/positioning impossible, which eliminates automation as an option. Third, for zebrafish embryo injection, the size of zebrafish embryos requires microneedles with a tip length of at least $600 \mu \mathrm{m}$ and outer diameter within $20 \mu \mathrm{m}$ (preferably within $10 \mu \mathrm{m}$ ) throughout the tip length in order to minimize cell damage. The injection needles also must be strong enough without buckling under hundreds of microNewton penetration forces during zebrafish embryo injection [29]. These requirements make the selection of MEMS-based solutions inappropriate.

Despite their relatively large size $(\sim 600 \mu \mathrm{m}$ or $\sim 1.2 \mathrm{~mm}$ including chorion), zebrafish embryos have a delicate structure and can be easily damaged. They are also highly deformable, making the automatic manipulation task difficult. Specific challenges in achieving automated, high-throughput zebrafish embryo injection include: i) the ability to quickly (i.e., seconds) immobilize a large number of zebrafish embryos into a regular pattern; ii) the ability to automatically and robustly identify cell structures for vision-based position control and account for size differences across embryos; and iii) the ability to coordinately control two motorized positioning devices (e.g., microrobots) to

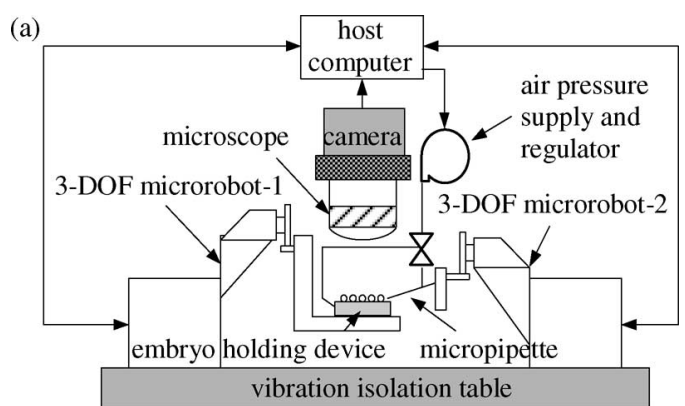

(b)

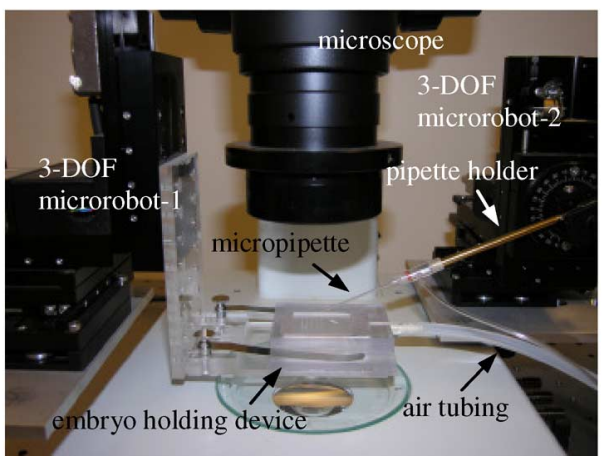

Fig. 2. Zebrafish embryo injection system. (a) Schematic diagram of the system architecture. (b) Picture of part of the system.

achieve robust, high-speed zebrafish embryo injection. In this paper, a recently developed system for zebrafish embryo injection is presented, featuring full automation, a high speed, fast sample immobilization, high success rates, and high survival rates.

\section{SYSTEM DESIGN}

\section{A. System Architecture}

The system, shown in Fig. 2, employs two three-degrees-offreedom microrobots (MP-285, Sutter) with a travel of $25 \mathrm{~mm}$ and a $0.04 \mu \mathrm{m}$ positioning resolution along each axis. Two motion control cards (NI PCI-6259) are mounted on a host computer (3.0 GHz CPU and 1 GB memory) where control algorithms and image processing algorithms operate. Visual feedback is provided through a CMOS camera (A601f, Basler) mounted on an optical microscope (SZX12, Olympus). An embryo holding device is attached to microrobot-1. A glass micropipette (TW120F-4, WPI), heated and pulled using a laser micropipette puller (Model 2000, Sutter), is connected to microrobot-2 via a micropipette holder (MPH412, WPI). A Venturi vacuum pump (UN816, KNF) provides negative pressure to immobilize embryos into regular patterns. A computer-controlled pico-injector (PLI-100, Harvard Apparatus) with a volume control resolution down to subpicoliter provides positive pressure for material deposition. To minimize vibration, all units except the host computer and pressure units are mounted on a vibration isolation table.

\section{B. Embryo Holding Device}

Placing individual zebrafish embryos into a regular pattern significantly simplifies the embryo searching/positioning task, 

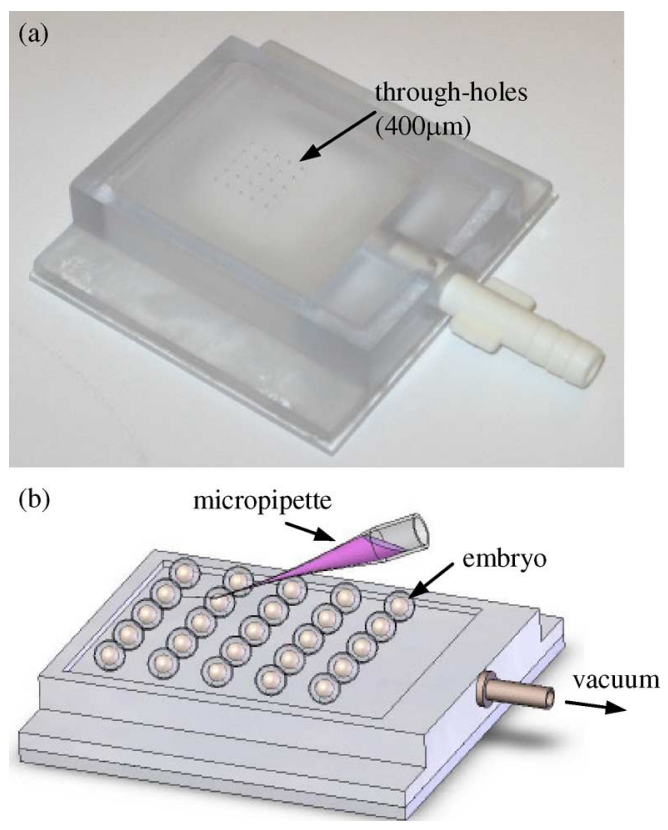

Fig. 3. Vacuum-based zebrafish embryo holding device. (a) Device picture. (b) Device schematic with embryos immobilized for injection.

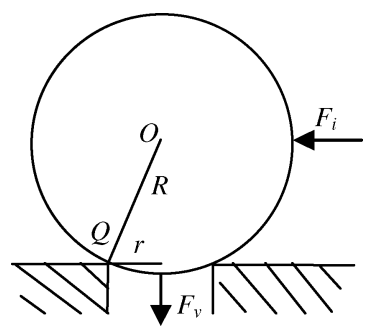

Fig. 4. Schematic for negative pressure estimation.

and therefore, increases injection speed. Vacuum-based immobilization was chosen since low vacuum levels prove effective and do not produce undesired biological complications for further cellular development. Vacuum-based immobilization is also advantageous, compared to techniques such as DEP (dielectropheresis) trapping [30] and laser trapping [31], in the simplicity of device construction.

Fig. 3 shows a vacuum-based embryo holding device for immobilizing individual zebrafish embryos. Evenly spaced through-holes $(\sim 400 \mu \mathrm{m})$ are connected to a vacuum source via a backside channel. Upon dispersing a batch of embryos onto the device, a sucking pressure enables each through-hole to trap a single embryo. The extra nontrapped embryos are flushed away from the device. Polycarbonate is used for constructing the embryo holding devices as it is optically transparent, biocompatible, inexpensive, and easy to machine or micromachine with laser ablation.

For guiding the selection of negative pressure levels, a brief analysis was conducted. As shown in Fig. 4, the pressure required to stabilize the embryo, which is assumed as a rigid object, can be obtained when equilibrium is maintained during injection. Let $F_{i}$ be the penetration force, $F_{v}$ the sucking force,
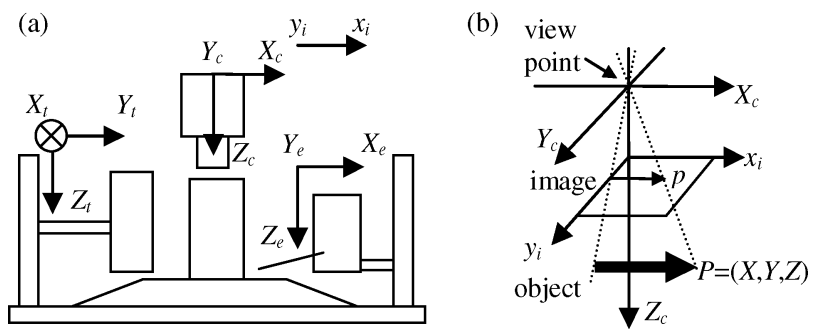

Fig. 5. (a) Coordinate frames of the system. (b) Image projection model relating the camera coordinate frame to the image plane.

\section{TABLE I}

SUMMARY OF COORDINATE FRAMES

\begin{tabular}{cl}
\hline Symbol & Coordinate frame \\
\hline$e$ & $\begin{array}{l}\text { End-effector coordinate frame } X_{e}-Y_{e}-Z_{e} \text { attached to } \\
\text { microrobot-2 that controls the motion of the injection mi- } \\
\text { cropipette }\end{array}$ \\
$\begin{array}{l}\text { Target coordinate frame } X_{t}-Y_{t}-Z_{t} \text { attached to microrobot-1 } \\
\text { that controls the motion of embryos }\end{array}$ \\
$\quad \begin{array}{l}\text { Camera coordinate frame } X_{c}-Y_{c}-Z_{c} \\
i\end{array} \quad$ Image plane $x_{i}-y_{i}$ (or $\left.x-y\right)$ \\
\hline
\end{tabular}

$O$ the center of the embryo, $Q$ the supporting point, and $R$ the radius of the embryo. The nominal negative pressure is

$$
P_{v}=\frac{\sqrt{R^{2}-r^{2}} F_{i}}{\left(\pi r^{3}\right)}
$$

Substituting $R=600 \mu \mathrm{m}, r=200 \mu \mathrm{m}, F_{i}=700 \mu \mathrm{N}$ [29] into (1) yields a nominally required pressure level of $4.7 \mathrm{InHg}$. In practice, a negative pressure of $2-7 \mathrm{InHg}$ proved effective in immobilizing zebrafish embryos of different strains without damaging the embryos.

\section{Coordinate Frames}

The coordinate frames of the system defined in Fig. 5(a) are summarized in Table I. A point $P=(X, Y, Z)$ in the camera frame $X_{c}-Y_{c}-Z_{c}$ is mapped to a point $p=(u, v)$ in the image plane $x-y$ via a scaled orthographic projection [Fig. 5(b)]

$$
\left[\begin{array}{cc}
s_{x} & 0 \\
0 & s_{y}
\end{array}\right]\left[\begin{array}{l}
u \\
v
\end{array}\right]=\left[\begin{array}{l}
X \\
Y
\end{array}\right]
$$

where $s_{x}$ and $s_{y}$ are scale factors (horizontal and vertical dimensions of a pixel). Calibration of $s_{x}$ and $s_{y}$ can be either manually, offline conducted or automatically, online conducted, as discussed in Section V.

\section{Injection Path Selection}

Upon the immobilization of embryos, the system sequentially conducts injection. As illustrated in Fig. 6, microrobot-1 can be controlled to travel along certain paths, out of which the shortest path should be selected in order to increase injection throughput. The labeled four paths, which represent the most efficient candidates for sequential batch injection, are considered here.

Denote by $\Delta X$ and $\Delta Y$ the pitch between adjacent throughholes along the $X_{t}$ and $Y_{t}$ directions, and denote by $m$ and $n$ the 


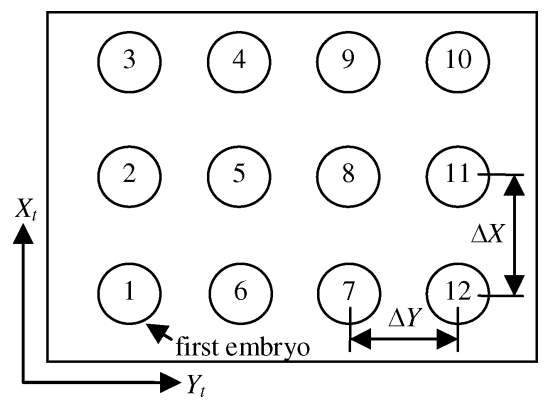

Fig. 6. Injection paths. Path-1: 1-2-3-4-5-6-7-8-9-10-11-12. Path-2: 1-6-712-11-8-5-2-3-4-9-10. Path-3: 1-2-3-4-9-10-11-12-7-6-5-8. Path-4: 1-6-7-12$11-10-9-4-3-2-5-8$.

number of embryos along the $X_{t}$ and $Y_{t}$ directions. The lengths of the four paths are

$d_{1}=n(m-1) \Delta X+(n-1) \Delta Y$

$d_{2}=m(n-1) \Delta Y+(m-1) \Delta X$

$d_{3}= \begin{cases}\left(m-1+C_{m}^{2}-C_{m-n+1}^{2}\right) \Delta X+C_{n}^{2} \Delta Y, & \text { if } m \geq n \\ \left(m-1+C_{m}^{2}\right) \Delta X+\left(C_{n}^{2}-C_{n-m}^{2}\right) \Delta Y, & \text { else }\end{cases}$

$d_{4}= \begin{cases}\left(n-1+C_{n}^{2}\right) \Delta Y+\left(C_{m}^{2}-C_{m-n}^{2}\right) \Delta X, & \text { if } m \geq n \\ \left(n-1+C_{n}^{2}-C_{n-m+1}^{2}\right) \Delta Y+C_{m}^{2} \Delta X, & \text { else }\end{cases}$

where $C_{x}^{y}$ is the binomial coefficient.

In the system reported in this paper, the embryo holding devices are designed to provide $\Delta X\langle\Delta Y, m>2$, and $n>2$, resulting in $d_{1}<d_{3}<d_{4}<d_{2}$. As path-1 is the shortest, it is selected as the injection sequence. Furthermore, in order to prevent crashing between the injection micropipette and embryos when moving from one embryo to another, $\Delta X$ and $\Delta Y$ were chosen to be $1.8 \mathrm{~mm}$ and $2.5 \mathrm{~mm}$, respectively.

\section{E. Volume Control}

Volume calibration is important for precisely depositing a specified amount of materials into individual cells such that dose effect can be investigated. Deionized (DI) water is used as an example in this section to describe the calibration of the relationship between injection volume, applied pressure, and pressure "on" time. A drop of DI water pushed out of the injection micropipette forms a sphere at the micropipette tip. Injection volume is then calculated by detecting the diameter of the sphere via a Hough transform.

For a micropipette tip with an inner diameter of $10 \mu \mathrm{m}$, Fig. 7 shows the relationship of injected volume versus pressure "on" time corresponding to two different injection pressure levels. By controlling the pressure "on" time, 3-nl materials were deposited into each zebrafish embryo in the experiments.

\section{Control Flow of Automated Cell Injection}

\section{A. Overall Sequence}

A batch of zebrafish embryos (e.g., 25), immobilized into a regular pattern on the embryo holding device, are placed on

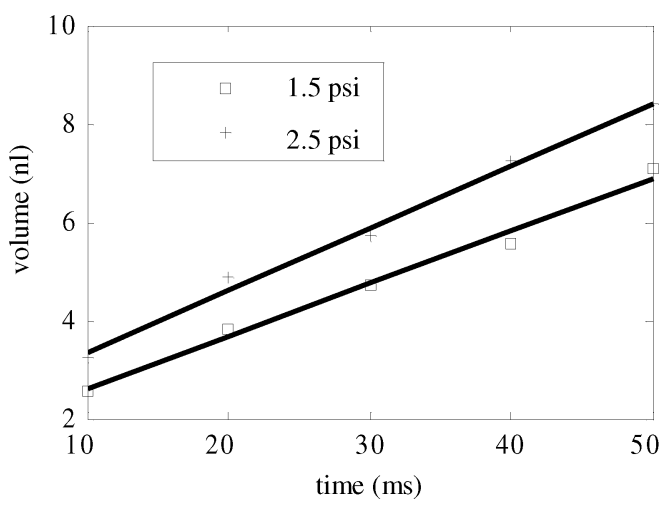

Fig. 7. Example data of injection volume calibration.

microrobot-1 under the microscope. Fully automated injection starts with vision-based contact detection [32] to determine the vertical positions of the micropipette tip and the top surface of the embryo holding device. An embryo is recognized and brought to the center of the field of view; simultaneously, the micropipette tip is moved by microrobot-2 to a switching point that is determined through the recognition of embryo structures. The micropipette tip penetrates the chorion and deposits materials at the desired location within the embryo. In the experiments presented in this paper, the deposition destination was chosen to be the cytoplasm center, where cytoplasm is defined as the combination of the yolk and the cell portion of a zebrafish embryo. Upon retreating out of the embryo, the micropipette tip is moved to a home position that is defined in Section III-C. In the mean time, the next embryo is brought into the field of view, the structures are recognized, and the injection process is repeated until all embryos in the batch are injected.

Throughout the process, microrobot- 1 does not produce vertical motion while microrobot- 2 is servoed along three axes, as shown in Fig. 8(a). Fig. 8(b) shows a sequence of pictures to illustrate the control flow. For positioning each embryo and controlling the motion of the injection micropipette, proportional-integral-derivative (PID) control is employed for controlling both microrobots that are operated in parallel whenever possible as indicated by “*” and “**” in Fig. 8(a). Parallel operation of the two microrobots is maximized to increase injection throughput.

\section{B. Microrobotic Control}

In the motion sequences described in Sections III-D and III-E, motion control of both microrobot- 1 and microrobot- 2 is based on position feedback of the microrobots (Fig. 9), following the PID control law. However, feature recognition from image processing is conducted to identify target positions $P_{d}$, making the system a "looking-then-moving" system. In order to determine the lateral components of $P_{d}$ from image feature recognition, coordinate transformations are performed between the image frame and the two microrobot frames.

Denote by ${ }^{t} P \in \Re^{2}$ the coordinates of point $P$ with respect to coordinate frame $t$. Denote by ${ }^{t} R_{c} \in \Re^{2 \times 2}$ the rotation matrix that represents the orientation of frame $c$ with respect to frame 


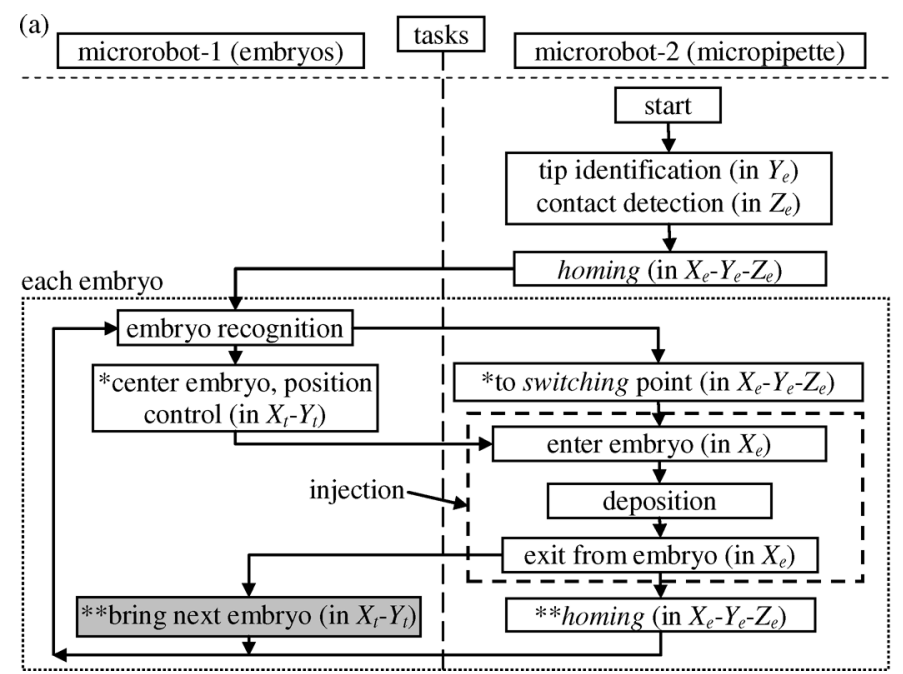

(b)

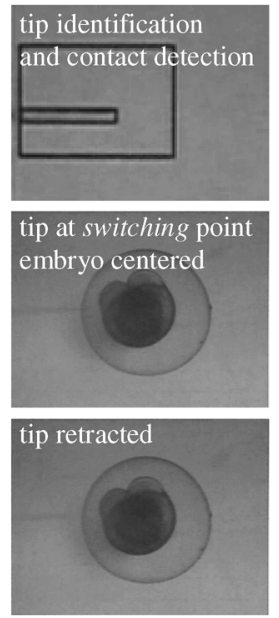

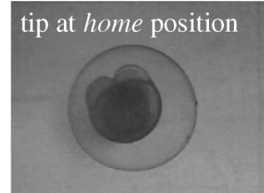
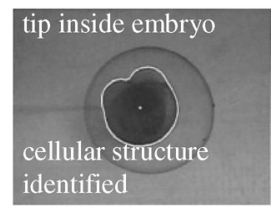

tip returning home

next embryo in view

Fig. 8. (a) Flow of automated cell injection. Except the shaded task, control of both microrobots is based on "looking-then-moving." “*” and “**” indicate parallel operation of the two microrobots. (b) A sequence of pictures illustrating the operation flow.

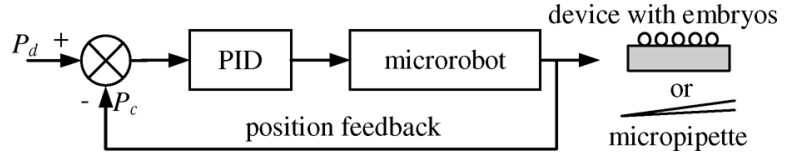

Fig. 9. Position control for both microrobots. Microrobot-1 controls the motion of embryos and microrobot- 2 controls the motion of the injection micropipette.

$t$. Denote by ${ }^{t} t_{c} \in \Re^{2}$ the location of the origin of frame $c$ with respect to frame $t$

$$
\begin{aligned}
{ }^{e} P & ={ }^{e} R_{c}{ }^{c} P+{ }^{e} t_{c} \\
{ }^{t} P & ={ }^{t} R_{c}{ }^{c} P+{ }^{t} t_{c} .
\end{aligned}
$$

Rewriting (2) as

$$
s^{i} p={ }^{c} P
$$

where

$$
s=\left[\begin{array}{cc}
s_{x} & 0 \\
0 & s_{y}
\end{array}\right] .
$$

Substituting (5) into (4) yields

$$
\begin{aligned}
& { }^{e} P={ }^{e} R_{c} s^{i} p+{ }^{e} t_{c} \\
& { }^{t} P={ }^{t} R_{c} s^{i} p+{ }^{t} t_{c} .
\end{aligned}
$$

According to the coordinate frames defined in Fig. 5(a)

$$
{ }^{e} R_{c}=\left[\begin{array}{ll}
1 & 0 \\
0 & 1
\end{array}\right], \quad{ }^{t} R_{c}=\left[\begin{array}{cc}
0 & 1 \\
-1 & 0
\end{array}\right] .
$$

With known $s$, the transformation between the image frame and the microrobot frames is uniquely established upon the determination of ${ }^{e} t_{c}$ and ${ }^{t} t_{c}$. When the micropipette tip is identified (Section III-C), ${ }^{e} t_{c}$ is solved from a determined pair of ${ }^{e} P$ and ${ }^{i} p$. When the cytoplasm center of an embryo in the field of view is recognized (Section III-E), ${ }^{t} t_{c}$ is solved from a determined pair of ${ }^{t} P$ and ${ }^{i} p$.

\section{Contact Detection and Home Position Determination}

It is desired for the micropipette tip to penetrate an embryo close to the center plane of the embryo (Fig. 10). Thus, it is imperative to determine the relative heights of the micropipette tip (controlled by microrobot-2) and the top surface of the embryo holding device (controlled by microrobot-1). A vision-based contact detection technique is developed [32] and briefly summarized here.

The micropipette, controlled by microrobot-2, first moves only along the $Y_{e}$ direction to identify the micropipette tip for subsequent contact detection. Based on boundary chain codes, the moving micropipette tip that stands out in the image is identified. Upon identification, the $x$-coordinate and $y$-coordinate in the image plane $x-y$ and the $X_{e}$-coordinate and $Y_{e}$-coordinate of the micropipette tip in the $X_{e}-Y_{e}-Z_{e}$ coordinate frame are determined and used to establish the transformation between the image frame and the $X_{e}-Y_{e}$ plane, according to (6). The $X_{e}$-coordinate and $Y_{e}$-coordinate in the $X_{e}-Y_{e}-Z_{e}$ coordinate frame are taken as the lateral components of the home position of the micropipette tip.

After the identification of the micropipette tip, the micropipette stops moving along the $Y_{e}$ direction. It is then controlled to move along the downward direction $\left(Z_{e}\right)$ at a constant speed to establish contact with the surface. After the establishment of the contact between the micropipette tip and the top surface of the embryo holding device, further vertical motion of the micropipette tip along the $Z_{e}$ direction results in lateral movement along the $X_{e}$ direction due to tip sliding. Before and after contact, the $x$-coordinates of the micropipette tip in the image plane $x-y$ result in a $\mathrm{V}$-shaped curve.

The peak of the V-shaped curve represents the contact position along the vertical direction between the micropipette tip and the top surface of the embryo holding device. Experimental results demonstrate that the computer vision-based contact detection technique is capable of achieving contact detection with an accuracy of $0.2 \mu \mathrm{m}$. Furthermore, 1000 experimental trials reveal that the technique is robust to variations in illumination intensity, microscopy magnification, and microrobot motion 


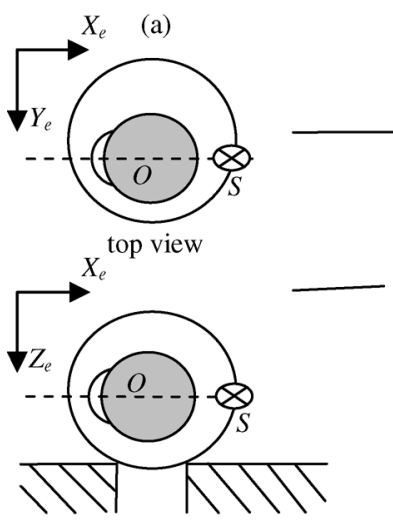

side view

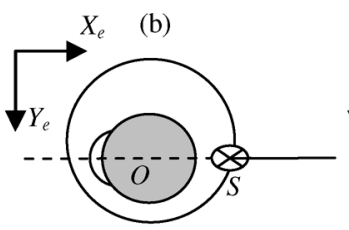

top view

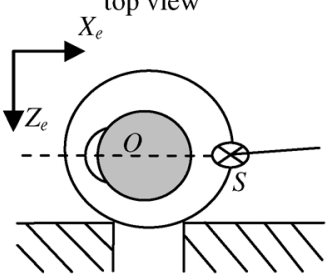

side view

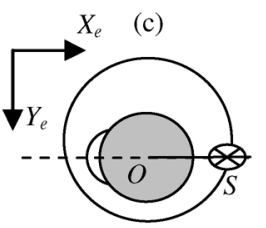

top view

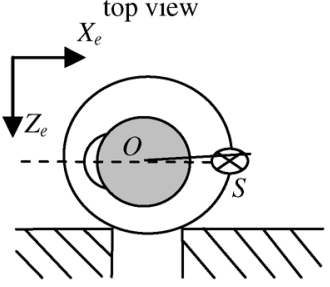

side view
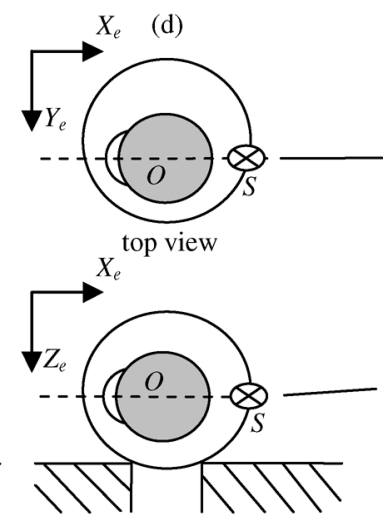

side view

Fig. 10. Micropipette motion sequence for injecting each embryo.

speed [32]. The entire contact detection process completes between 6 and $10 \mathrm{~s}$.

The $Z_{e}$-coordinate of the home position of the micropipette tip is set at $1.4 \mathrm{~mm}$, which is slightly greater than the embryo diameter, above the contact position. Thus, the home position of the micropipette tip in the $X_{e}-Y_{e}-Z_{e}$ coordinate frame is automatically determined. While microrobot- 1 brings the next embryo into the field of view, microrobot- 2 moves the micropipette tip upwards and laterally to the home position (i.e., homing in Fig. 8) to prevent the micropipette tip from crashing into embryos.

\section{Micropipette Motion Sequence}

As shown in Fig. 10, the two principal planes, which cross the cytoplasm center $O$ and are, respectively, parallel to the $X_{e}-Z_{e}$ plane and to the $X_{e}-Y_{e}$ plane, overlap at two points. The point closer to the micropipette tip is referred to as the switching point, $S$. Experimentally, the lateral coordinates of $S$ and embryo radius are determined by image processing (Section IV-B). The $Z_{e}$-coordinate of $S$ is determined as a value equal to the radius of the embryo above the contact position.

The motion sequence of the micropipette tip, controlled by microrobot- 2 for injecting an embryo includes the following steps. 1) To move from the home position to the switching point, simultaneously along all three axes [Fig. 10(b)]. 2) To penetrate the chorion and cytoplasm membrane and to move to the cytoplasm center (deposition destination) along the $X_{e}$ direction only [Fig. 10(c)] at a speed of $1.5 \mathrm{~mm} / \mathrm{s}$. Upon reaching the cytoplasm center, the computer-controlled pico-injector is triggered, and a pre-specified amount of materials is deposited. 3) To retreat from the cytoplasm center beyond the switching point along the $X_{e}$ direction only [Fig. 10(d)] at a speed of $2.9 \mathrm{~mm} / \mathrm{s}$. 4) To return to the home position. When the next embryo is brought into the field of view, the injection process repeats [Fig. 10(a)]. The criterion for determining the injection and retraction speeds is to minimize injection-induced cell lysis.

\section{E. Embryo Motion Sequence}

The position of the embryos sitting on the embryo holding device is controlled by microrobot-1. When moving from one embryo to the next embryo, microrobot-1 first brings the next embryo into the field of view according to the known pitch $(\Delta X$ or $\Delta Y$ ) between adjacent through-holes of the embryo holding device. The recognized cytoplasm center of the embryo, ${ }^{i} p$ and the corresponding position of microrobot- $1,{ }^{t} P$ are used to determine the transformation between the image frame and the $X_{t}-Y_{t}$ plane, according to (7). The target position, $P_{d}$ in the $X_{t}-Y_{t}$ plane for microrobot-1 is then obtained from (7) and the image center position. Following PID control as described in Fig. 9, microrobot-1 brings the embryo to the image center.

\section{RECOGNITION OF EMBRYO STRUCTURES}

A zebrafish embryo consists of the chorion, cytoplasm membrane, the yolk, and the cell portion (in this paper, the yolk and cell portion combined are referred to as cytoplasm). The purpose of recognizing detailed embryo structures is for the determination of the deposition destination to guarantee a high reproducibility.

In the experiments presented in this paper, the identified cytoplasm center [Fig. 11(c)] was chosen as the deposition destination. However, the recognition algorithm allows for choosing a different destination, for example, closer to the yolk/cell interface to facilitate the diffusion of injected molecules into the cell portion. The recognition of detailed embryo structures takes $45 \mathrm{~ms}$ on the host computer.

\section{A. Pre-Processing}

Pre-processing is conducted to obtain de-noised binary images. An image is first convolved with a low-pass Gaussian filter for noise suppression. The gray-level image is then binarized to a black-white image using an adaptive thresholding method, in which a local threshold for each pixel $(u, v)$ is set to be the mean value of its $a \times b$ neighbors, i.e.,

$$
T(u, v)=\frac{1}{a \cdot b} \sum_{i=-a / 2}^{a / 2} \sum_{j=-b / 2}^{b / 2} f(u+i, v+j) .
$$

The binary image is eroded to remove small areas that represent spurious features and then, dilated to connect broken segments that originally belong to one object. An example after pre-processing is shown in Fig. 11(b). 

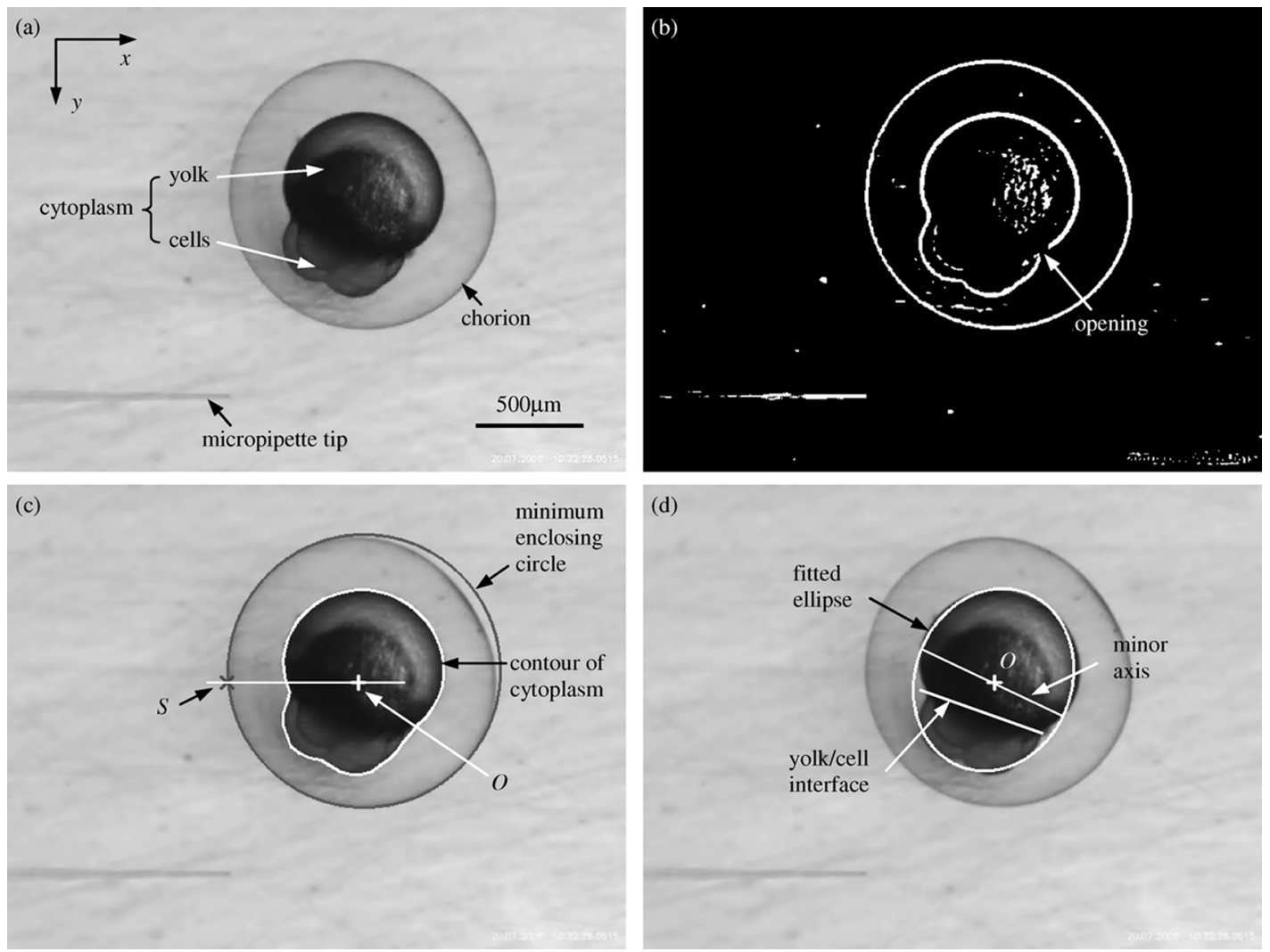

Fig. 11. Recognition of zebrafish embryo structures. Image under $2.5 \times$. (a) Zebrafish embryo. (b) After pre-processing. (c) Recognized chorion, cytoplasm center, and switching point. (d) Distinguished yolk and cell portion.

\section{B. Recognition of Chorion and Cytoplasm}

Of the connected objects in the binary image, the one with the maximum area is recognized as the chorion. In Fig. 11(c), the chorion is enclosed by its minimum enclosing circle.

The second largest object in the images is the cytoplasm, the boundary of which is represented by a chain code contour. The boundary of the cytoplasm is often not fully connected (Fig. 11(b)); however, a fully closed contour is important for the recognition of detailed cytoplasm structures including the yolk, the cell portion, and the yolk-cell interface. Thus, a convex hull [33] of the contour is used for further processing.

A region $R$ is convex if and only if for any two points $x_{1}$, $x_{2} \in R$, the complete line segment $x_{1} x_{2}$ is inside the region $R$. The convex hull $H$ of a region $R$ is the smallest convex region that satisfies the condition $R \subseteq H$. The constructed convex hull of the cytoplasm contour is used to provide initial positions for subsequent "snake" tracking [34]. The closed cytoplasm contour resulting from snake tracking is shown in Fig. 11(c). The centroid of the contour, $O$ is recognized as the cytoplasm center. The switching point, $S$ is then determined as the intersect point of the minimum enclosing circle and the horizontal line passing through the cytoplasm center.

\section{Cytoplasm Structure Recognition}

Distinguishing the yolk from the cell portion provides the flexibility for choosing a desired destination (e.g., yolk-cell interface) for material deposition other than the cytoplasm center.

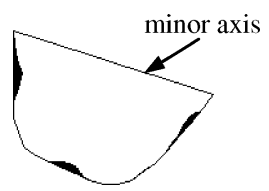

Fig. 12. Convexity defect (shaded area).

In order to determine which end of the cytoplasm is the cell portion, the cytoplasm contour after snake tracking is fitted into an ellipse using a least squares method. The cytoplasm contour is then intercepted into two parts by the minor axis of the fitted ellipse.

Define the area difference between a contour and its convex hull as the convexity defect (Fig. 12). Since the yolk always has a much more circular shape than the cell portion (i.e., smaller convexity defect), the part of the cytoplasm contour with a greater defect is recognized as the cell part. Subsequently, the line connecting the centroids of the two convexity defects close to the minor axis is recognized as the yolk/cell interface [Fig. 11(d)].

\section{Online Pixel Size CAlibration}

The operation flow described in Fig. 8 requires a prior knowledge of pixel sizes that are obtained through offline pixel size calibration. As pixel sizes vary with different microscope objectives, couplers, and cameras used for imaging, it is desired to eliminate the imaging-hardware dependence by conducting online calibration. 


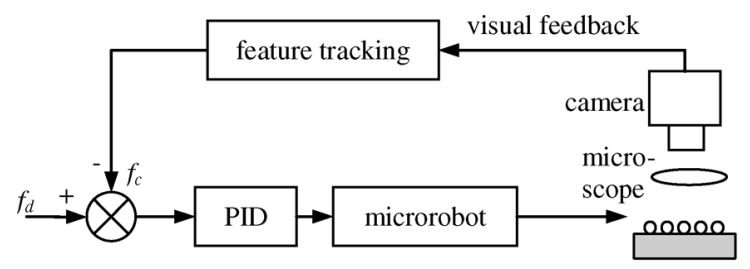

device with embryos

Fig. 13. Image-based visual servoing of microrobot-1 to position the first embryo for online pixel size calibration.

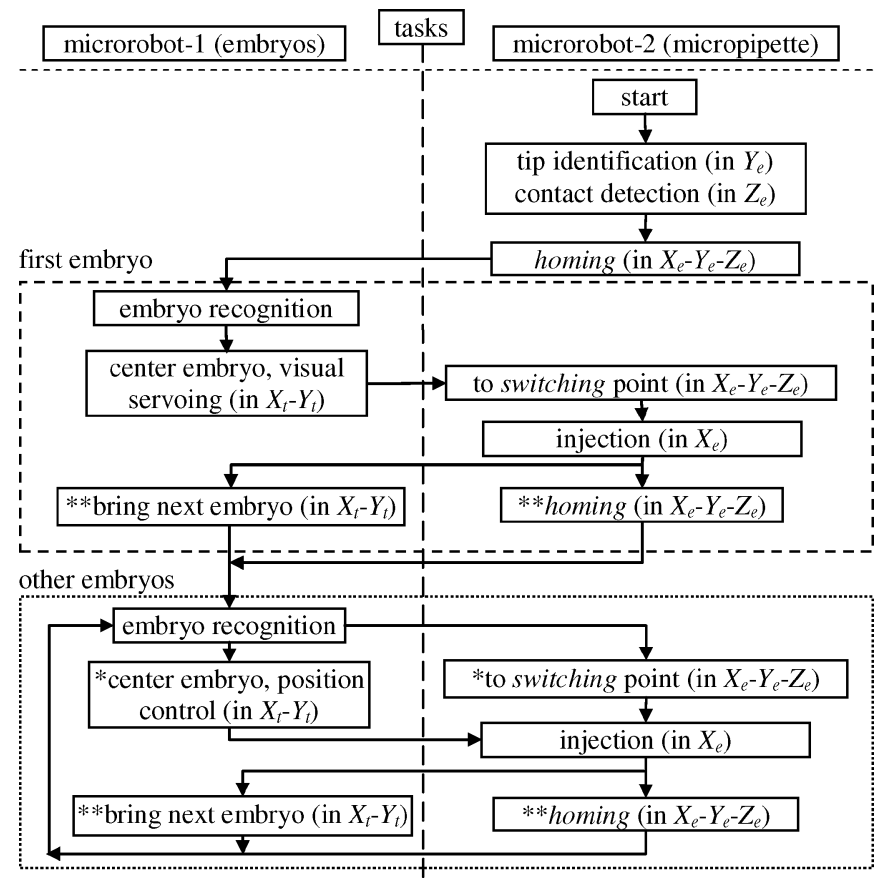

Fig. 14. Modified operation flow integrating online pixel size calibration. “ $*$ " and "*** indicate parallel operation of the two microrobots.

The system permits online calibration when positioning the first embryo within a batch. Microrobot-1 brings the first embryo to the image center according to image-based visual servoing (Fig. 13), while the other embryos within the batch are still brought to the image center by position control. Correspondingly, the injection flow is modified as Fig. 14.

\section{A. Image-Based Visual Servoing}

For a point $P=(X, Y, Z)$ rigidly attached to the target coordinate frame $X_{t}-Y_{t^{-}} Z_{t}$, its translational velocities with respect to the camera frame $X_{c}-Y_{c}-Z_{c}$ are

$$
{ }^{c} T_{t}=\left[T_{x}, T_{y}, T_{z}\right]^{\mathrm{T}} \text {. }
$$

Differentiating (2) yields

$$
\left[\begin{array}{cc}
s_{x} & 0 \\
0 & s_{y}
\end{array}\right]\left[\begin{array}{c}
\dot{u} \\
\dot{v}
\end{array}\right]=\left[\begin{array}{c}
\dot{X} \\
\dot{Y}
\end{array}\right]=\left[\begin{array}{lll}
1 & 0 & 0 \\
0 & 1 & 0
\end{array}\right]{ }^{c} T_{t} .
$$

According to the coordinate frames defined in Fig. 5(a)

$$
{ }^{c} T_{t}=\left[\begin{array}{rrr}
0 & -1 & 0 \\
1 & 0 & 0 \\
0 & 0 & 1
\end{array}\right]{ }^{t} T_{t}
$$

where ${ }^{t} T_{t}$ are translational velocities of the point $P$ in the target frame $X_{t}-Y_{t}-Z_{t}$.

Substituting (12) into (11) yields

$$
\left[\begin{array}{l}
\dot{u} \\
\dot{v}
\end{array}\right]=J \cdot{ }^{t} T_{t}, \quad J=\left[\begin{array}{ccc}
0 & -\frac{1}{s} x & 0 \\
\frac{1}{s} y & 0 & 0
\end{array}\right]
$$

that associates the change rate of an image feature with the translational velocities in the target frame via an image Jacobian matrix $J$.

Since $s_{x}$ and $s_{y}$ are unknown and yet to be online calibrated, the translational velocities of microrobot- 1 along $X_{t}$ and $Y_{t}$ are not solvable through the image Jacobian matrix. Instead, the translational velocities are regulated by the PID control law

$$
{ }^{t} T_{t}=K_{P} e(t)+K_{I} \sum e(t)+K_{D}[e(t)-e(t-1)]
$$

where $e(t)=f_{d}-f_{c}(t), f_{c}$ is the cytoplasm center, and $f_{d}$ is the image center (Fig. 13).

Integrating (13) produces

$$
\left[\begin{array}{l}
d u \\
d v
\end{array}\right]=\left[\begin{array}{c}
\frac{-d Y_{t}}{s_{x}} \\
\frac{d X_{t}}{s_{y}}
\end{array}\right]
$$

where $(d u, d v)$ represent displacements from the initial cytoplasm center to the image center in the image plane, and $\left(d X_{t}, d Y_{t}\right)$ represent displacements of microrobot-1 in the visual servoing process. Pixel size is thus calibrated as

$$
s_{x}=\left|\frac{d Y_{t}}{d u}\right|, \quad s_{y}=\left|\frac{d X_{t}}{d v}\right| .
$$

\section{B. Feature Tracking and Pixel Size Calibration}

In order to obtain feedback for image-based visual servoing of the first embryo, a sum-of-squared-differences (SSD) tracking algorithm [35] is employed to track the cytoplasm center $f_{c}$ that is identified from the recognition process described in Section IV.

The basic assumption of SSD tracking is that frame intensity $I(u, v)$ in a sequence of images does not change rapidly between successive images. In implementing the algorithm, a template $T$ of $20 \times 20$ pixels was acquired around the feature point [i.e., the cytoplasm center $\left.\left(u_{c}, v_{c}\right)\right]$. The SSD correlation measure is calculated for each possible displacement $(\Delta u, \Delta v)$ within a search window in the updated image

$$
\begin{aligned}
& \operatorname{SSD}(\Delta u, \Delta v) \\
& =\sum_{i, j \in N}\left[I\left(u_{c}+\Delta u+i, v_{c}+\Delta v+j\right)-T\left(u_{c}+i, v_{c}+j\right)\right]^{2} .
\end{aligned}
$$

The distance $(\Delta u, \Delta v)$ having the minimum SSD measure shown in (17) is taken to be the displacement of the feature. The amount of processing depends greatly on the template size and the size of the search window. A large template increases robustness while a large search window helps handle large displacements, provided frames of images can be processed in real time. In the implementation, a search window of $80 \times 80$ pixels was acquired.

Trials for online calibration were repeated 100 times. In each trial, the embryo was visually servoed from the same starting 


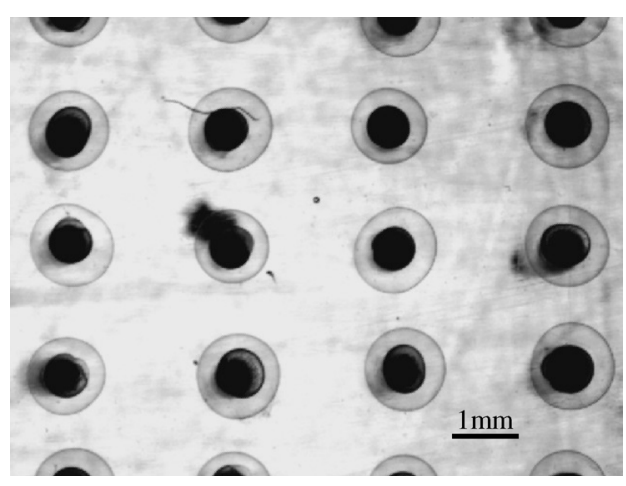

Fig. 15. Array of immobilized embryos on the embryo holding device.

position to the image center. To quantify the error in SSD feature tracking, the cytoplasm center of the first embryo was recognized at the end of visual servoing and was compared to the position located from SSD tracking. The standard deviation of such determined distances was 0.72 pixel for the 100 trials at a magnification of $2.5 \times$, demonstrating a high accuracy of cytoplasm center tracking.

When microrobot- 1 reaches steady state (i.e., reaching the image center, $f_{d}$ ), both pixel displacements in the image plane $x-y$ and microrobot displacements in the $X_{t}-Y_{t}$ plane are obtained. Thus, pixel sizes are online calibrated according to (16). The online calibrated pixel sizes from the 100 trials were $s_{x}=$ $s_{y}=3.96 \pm 0.1 \mu \mathrm{m}$ for a magnification of $2.5 \times$. The small standard deviation demonstrates the system's satisfactory performance in online calibration.

\section{EXPERIMENTAL RESULTS AND DISCUSSION}

\section{A. Materials}

The zebrafish embryos used in the injection experiments were collected in The Hospital for Sick Children (Toronto, Canada) with standard embryo preparation procedures [23]. The outbred zebrafish embryos, which were not de-chorionized, were cultured in embryo media that contained 101 reverse osmosis water, $3 \mathrm{~g}$ instant ocean salt mix, and $10 \mathrm{ml}$ methylene blue solution ( stock $=1 \mathrm{gm} / \mathrm{l})$.

Fluorescent dyes (Rhodamine B, $100 \mu \mathrm{M}$ ) were used as testing injection materials, which allow for visually inspecting the effectiveness of automated injection.

\section{B. Results}

1) Embryo Patterning: The collected embryos were spread on the surface of the embryo holding device together with the media. Applied negative pressure immobilized individual embryos on top of each through-hole. The extra embryos were flushed off the embryo holding device. Fig. 15 shows an array of immobilized embryos, taken under a low magnification of $0.7 \times$. The process of embryo patterning is manually assisted, taking approximately 6-12 s. Regularly patterned embryos eliminate random searching and increase injection speed.

2) System Performance Evaluation: The automated system continuously injected a total of 250 zebrafish embryos with fluorescent dyes, demonstrating an operation speed of 15 embryos/
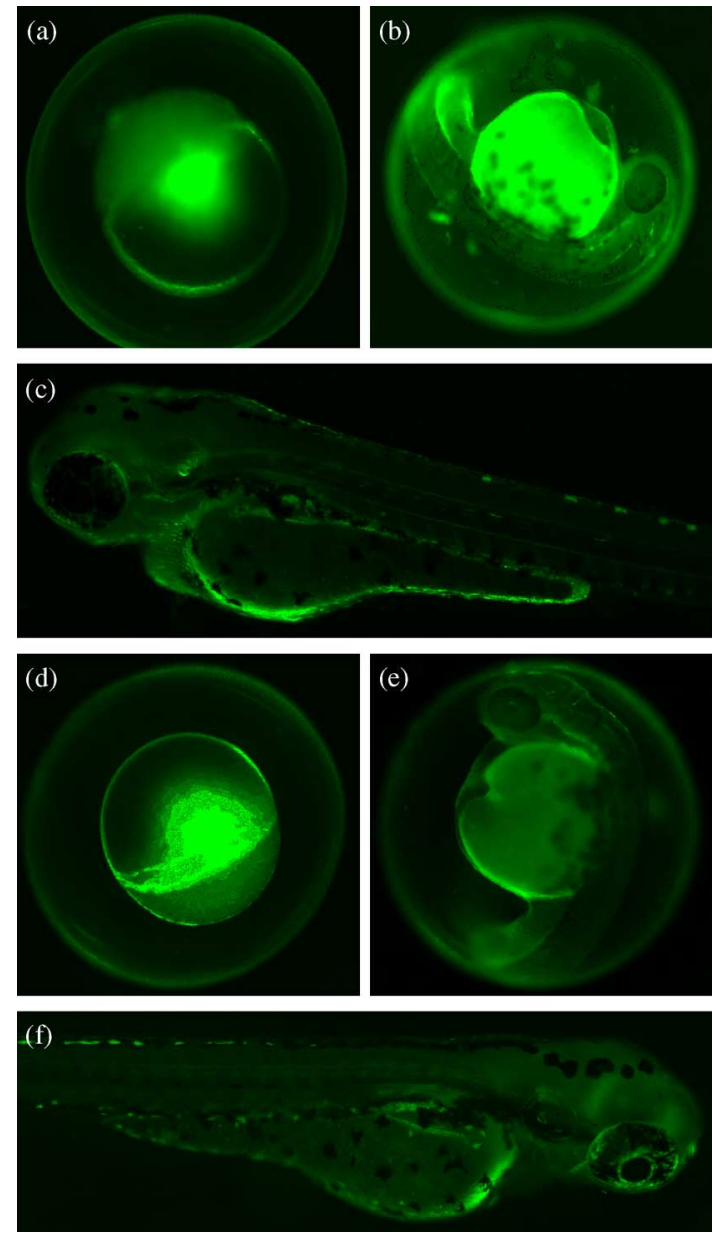

Fig. 16. Development of wild-type zebrafish embryos injected with fluorescent dyes. (a), (b), (c) for sample-1 and (d), (e), (f) for sample-2. (a), (d) Right after injection. (b), (e) $24 \mathrm{~h}$ after injection. (c), (f) $48 \mathrm{~h}$ after injection.

min. This injection speed is comparable to that of a highly proficient injection technician. However, the system is advantageous in that it is not only free from fatigue, but also provides unparalleled reproducibility.

The injected embryos were cultured at $32{ }^{\circ} \mathrm{C}$ and inspected under a fluorescence microscope (IX81, Olympus). The embryos were excited by $540 \mathrm{~nm}$ laser light and observed through a TRITC filter set. Visual inspection was conducted right after injection, $24 \mathrm{~h}$ after injection, and $48 \mathrm{~h}$ after injection. Fig. 16 shows two injected embryos and their subsequent development. The deposited fluorescent dyes (high-brightness area) can be clearly observed at each developmental stage.

In order to quantitatively evaluate the performance of the automated injection system, two measures were defined. 1) Success rate: This measure is defined as the ratio between the number of embryos with fluorescent dyes successfully deposited in the cytoplasm center and the total number of injected embryos. Essentially, this measure represents the reliability and the reproducibility of the system. Visual inspection demonstrated that the success rate of the 250 injected embryos was $100 \%$. 2) Survival rate: This measure is defined as the ratio between the number of injected embryos that are capable of developing into larva and the total number of embryos injected, 
essentially representing the severity and frequency of cell damage due to injection. Based on the 250 injected zebrafish embryos, the injection system produced a satisfactory survival rate of $98 \%$.

\section{Discussion}

The achieved survival rate of $98 \%$ is consistent with the best survival rate achieved by proficient injection technicians. However, the system is immune from large variations in the survival rate that can reach as low as $50 \%-70 \%$ in manual operation, due to technician fatigue and proficiency differences across technicians. Furthermore, the operation speed of the automated system (15 embryos with unremoved chorion per minute) compares favorably with manual injection speed, which is estimated by injection technicians to be $8-20$ embryos/min. Importantly, the embryo holding device and technique developed in this research permit the completion of immobilizing zebrafish embryos into regular patterns within seconds while manually pushing embryos into agarose trenches, as in the state-of-the-art zebrafish embryo injection, would cost minutes.

The high survival rate results from efforts on minimizing embryo lysis. Inappropriate selections of injection and retraction speeds of the injection micropipette, the use of micropipette tips of large diameters (e.g., $20 \mu \mathrm{m}$ ), and too high a suction pressure can all lead to a high frequency of lysis, resulting in low survival rates. The parameters reported in this paper (injection speed: $1.5 \mathrm{~mm} / \mathrm{s}$; retraction speed: $2.9 \mathrm{~mm} / \mathrm{s}$; suction pressure: 2-7 InHg) were determined from trials on another 300 zebrafish embryos during system development.

The achieved success rate of $100 \%$ demonstrates that the automated system is capable of repeatedly depositing materials at a desired destination inside zebrafish embryos for each operation. This high reproducibility, resulting from the recognition of embryo structures and precise motion control, is unparalleled by manual operation in which materials are deposited at random locations inside embryos. The ability of precisely depositing materials at a desired location in a highly reproducible manner has important implications. The elimination of length variations in diffusion paths would make the results of molecule or drug compound screening more countable. The system also allows the selection of desired deposition destinations other than the cytoplasm center, for example, closer to the interface between the yolk and the cell portion or directly inside the cells.

Further enhancement of the automated system will integrate an injection pressure monitoring mechanism for detecting micropipette clogging from embryo debris accumulation. As micropipette clogging occurs gradually, leading to gradual decreases of material deposition volume, the integration of a pressure sensor and a closed-loop injection pressure compensation module will improve deposition volume control, which is critical for more stringently investigating dose responses to biomolecules or drug compounds.

\section{CONCLUSION}

Based on the technology of computer vision and microrobotics, the high-throughput automated cell injection system experimentally demonstrated the capability of injecting 15 zebrafish embryos per minute with a $100 \%$ success rate and a
$98 \%$ survival rate. The vacuum-based embryo holding device is capable of immobilizing a large number of embryos into regular patterns within seconds, dramatically shortening the sample preparation process. The recognition of embryo structures and precise motion control enable the automated system to precisely deposit a pre-specified amount of materials at a desired destination within the embryo. The application of the automated cell injection system, which features a high speed, a high success rate, and a high survival rate, to biological and pharmaceutical research for timely injecting materials into a larger number of cells will facilitate biomolecule or drug compound screening. Despite size and property differences among different cell lines, the sample preparation technique and microrobotic control method are applicable to other injection applications such as the injection of mouse oocytes/embryos and other types of suspended cells.

\section{ACKNOWLEDGMENT}

The authors thank Prof. B. Ciruna at the Hospital for Sick Children and Prof. A. Bruce in the Zoology Department at the University of Toronto for providing zebrafish embryos and constructive discussions.

\section{REFERENCES}

[1] W. C. Shen and H. J. Ryser, "Selective killing of fc-receptor-bearing tumor cells through endocytosis of a drug-carrying immune complex," Proc. Nat. Acad. Sci., vol. 81, no. 5, pp. 1445-1447, 1984.

[2] V. Khemka, D. See, J. See, J. Chang, S. Chou, and J. Tilles, "The capacity of a combined liposomal hepatitis $\mathrm{B}$ and $\mathrm{C}$ vaccine to stimulate humoral and cellular responses in mice," Viral. Immunol., vol. 11, no. 2, pp. 73-78, 1998.

[3] G. C. Fanning, "RNA as a target for host defense and anti-hiv drugs," Curr. Drug. Target., vol. 7, no. 12, pp. 1607-1613, 2006.

[4] G. L. Beretta, P. Perego, and F. Zunino, "Mechanisms of cellular resistance to camptothecins," Curr. Med. Chem., vol. 13, no. 27, pp. 3291-3305, 2006.

[5] A. Kalota, S. E. Shetzline, and A. Gewirtz, "Progress in the development of nucleic acid therapeutics for cancer," Cancer Biol. Ther., vol. 3, no. 1, pp. 4-12, Jan. 2004.

[6] J. Sundaram, B. R. Mellein, and S. Mitragotri, "An experimental and theoretical analysis of ultrasound-induced permeabilization of cell membranes," Biophys. J., vol. 87, no. 2, pp. 1013-1033, 2004.

[7] M. P. Rols, "Electropermeabilization, a physical method for the delivery of therapeutic molecules into cells," Biochim. Biophys. Acta., vol. 1758 , no. 3, pp. 423-428, 2006

[8] J. W. Park, "Liposome-based drug delivery in breast cancer treatment," Breast Cancer Res., vol. 4, pp. 95-99, 2002.

[9] M. D. Chavanpatil, A. Khdair, and J. Panyam, "Nanoparticles for cellular drug delivery: Mechanisms and factors influencing delivery," $J$. Nanosci. Nanotechnol., vol. 6, pp. 2651-2663, 2006.

[10] Y. Sun and B. J. Nelson, "Biological cell injection using an autonomous microrobotic system," Int. J. Robot. Res., vol. 21, no. 10-11, pp. 861-868, 2002.

[11] J. E. Celis, "Microinjection of somatic cells with micropipettes: Comparison with other transfer techniques," Biochem. J., vol. 223, no. 2, pp. 281-291, 1984.

[12] Z. Zhao, Y. Cao, M. Li, and A. Meng, "Double-stranded RNA injection produces nonspecific defects in zebrafish," Dev. Biol., vol. 229, no. 1, pp. 215-223, Jan. 2001.

[13] P. Scherp and K. H. Hasenstein, "Microinjection-a tool to study gravitropism," Space Res., vol. 31, no. 10, pp. 2221-2227, Mar. 2003.

[14] N. D. Tran, X. Liu, Z. Yan, D. Abbote, Q. Jiang, E. B. Kmiec, C. D. Sigmund, and J. F. Engelhardt, "Efficiency of chimeraplast gene targeting by direct nuclear injection using a GFP recovery assay," Mol. Ther., vol. 7, no. 2, pp. 248-253, Feb. 2003.

[15] E. Gahtan and D. M. O'Malley, "Visually guided injection of identified reticulospinal neurons in zebrafish: A survey of spinal arborization patterns," J. Comp. Neurol., vol. 459, no. 2, pp. 186-200, Apr. 2003. 
[16] R. King, "Gene delivery to mammalian cells by microinjection," Methods Mol. Biol., vol. 245, pp. 167-174, 2004.

[17] R. Kumar, A. Kapoor, and R. H. Taylor, "Preliminary experiments in robot/human cooperative microinjection," in Proc. IEEE Int. Conf. Intelligent Robots Syst. (IROS'2003), 2003, pp. 3186-3191.

[18] H. Matsuoka, T. Komazaki, Y. Mukai, M. Shibusawa, H. Akane, A. Chaki, N. Uetake, and M. Saito, "High throughput easy microinjection with a single-cell manipulation supporting robot," J. Biotechnol., vol. 116, no. 2, pp. 185-194, 2005.

[19] K. Kobayashi, K. Kato, M. Saga, M. Yamane, C. Rothman, and S. Ogawa, "Subzonal insemination of a single mouse spermatozoon with a personal computer-controlled micromanipulation system," Mol. Reproduct. Dev., vol. 33, pp. 81-88, 1992.

[20] L. Mattos, E. Grant, R. Thresher, and K. Kluckman, "New developments towards automated blastocyst microinjections," in Proc. IEEE Int. Conf. Robotics Autom. (ICRA'2007), 2007, pp. 1924-1929.

[21] A. Pillarisetti, M. Pekarev, A. D. Brooks, and J. P. Desai, "Evaluating the role of force feedback for biomanipulation tasks," in Proc. Symp. Haptic Interfaces For Virtual Environ. Teleoperator Syst. (HAPTICS'2006), 2006, pp. 11-18.

[22] S. Zappe, M. Fish, M. P. Scott, and O. Solgaard, "Automated mems-based drosophila embryo injection system for high-throughput rnai screens," Lap Chip., vol. 6, no. 8, pp. 1012-1019, Aug. 2006.

[23] M. Westerfield, The Zebrafish Book. Eugene, OR: Univ. Oregon Press, 1995.

[24] S. Hashmi, P. Ling, G. Hashmi, M. Reed, R. Gaugler, and W. Trimmer, "Genetic transformation of nematodes using arrays of micromechanical piercing structures," BioTechniques, vol. 19, pp. 766-770, 1995.

[25] K. Chun, G. Hashiguchi, H. Toshiyoshi, H. Fujita, Y. Kikuchi, J. Ishikawa, Y. Murakami, and E. Tamiya, "An array of hollow micro-capillaries for the controlled injection of genetic materials into animal/plant cells," in Proc. IEEE Int. Conf. Micro Electro Mech. Syst. (MEMS'1999), 1999, pp. 406-411.

[26] S. B. Leighton and M. J. Brownstein, "Array-Type multiple cell injector," U.S. 5262128, Nov. 1993

[27] R. O. Ginaven and D. Facciotti, "Needle array and method of introducing biological substances into living cells using the needle array," U.S. 5457041, Oct. 1995.

[28] Y. Yeshurun, "Systems and methods for the transport of fluids through a biological barrier and production techniques for such systems," U.S. 6558361, May 2003.

[29] X. Y. Liu, Y. Sun, W. H. Wang, and B. M. Lansdorp, "Vision-based cellular force measurement using an elastic microfabricated device," $J$. Micromech. Microeng., 2007.

[30] T. P. Hunt, H. Lee, and R. M. Westervelt, "Addressable micropost array for the dielectrophoretic manipulation of particle in fluid," App. Phy. Lett., vol. 85, no. 26, pp. 6421-6423, 2004.

[31] A. Ashkin, K. M. Dziedzic, and T. Yamane, "Optical trapping and manipulation of single cells using infrared laser beams," Nature, vol. 330, pp. 769-771, 1987.

[32] W. H. Wang, X. Y. Liu, and Y. Sun, "Contact detection in microrobotic manipulation,” Int. J. Robot. Res., vol. 26, pp. 821-828, 2007.

[33] R. Gonzalez and R. Woods, Digital Image Processing, 2nd ed. Upper Saddle River, NJ: Prentice-Hall, 2002.

[34] C. Y. Xu and J. L. Prince, "Snakes, shapes, and gradient vector flow," IEEE Trans. Image Process., vol. 7, no. 3, pp. 359-369, Mar. 1998
[35] K. Nickels and S. Hutchinson, "Estimating uncertainty in ssd-based feature tracking," Image Vis. Comput., vol. 20, no. 1, pp. 47-58, Jan. 1, 2002.

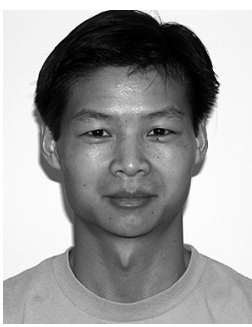

Wenhui H. Wang (M'06) received the B.S. degree in mechanical and electronic engineering and the M.S. degree from the Beijing Institute of Technology, Beijing, China, in 1998 and 2001,respectively, and the $\mathrm{Ph} . \mathrm{D}$. degree in mechanical engineering from the $\mathrm{Na}-$ tional University of Singapore in 2005.

He held a Research Associate position at the University of Toronto, Toronto, ON, Canada, before joining the faculty of the University of Canterbury, where he is Lecturer with the Mechanical Engineering Department. His research areas include computer vision, micromanipulation, bio-device and instrumentations, visual servo control, and manufacturing process monitoring.

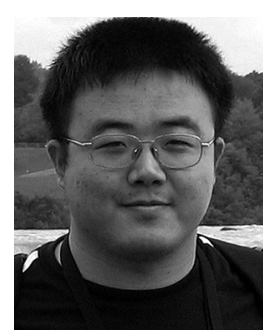

Xinyu Y. Liu (S'06) received the B.S. and M.S degrees in mechanical engineering from Harbin Institute of Technology, Harbin, China, in 2002 and 2004, respectively. He is now working towards the Ph.D. degree in the Department of Mechanical and Industrial Engineering, University of Toronto, Toronto, ON, Canada.

His research area is microrobotic cell manipulation.

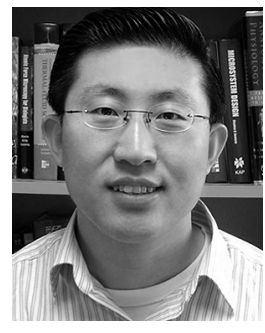

Yu Sun (S'01-M'03-SM'07) received the B.S degree in electrical engineering from Dalian University of Technology, China, in 1996, the M.S. degree from the Institute of Automation, Chinese Academy of Sciences, Beijing, China, in 1999, and the M.S. degree in electrical engineering and the $\mathrm{Ph} . \mathrm{D}$. degree in mechanical engineering from the University of Minnesota, Minneapolis, in 2001 and 2003, respectively.

He held a Research Scientist position at the Swiss Federal Institute of Technology (ETH-Zürich) before joining the faculty of the University of Toronto where he is Assistant Professor of Mechanical and Industrial Engineering Department and is jointly appointed in the Institute of Biomaterials and Biomedical Engineering and Electrical and Computer Engineering Department. He established and directs the Advanced Micro and Nanosystems Laboratory at Toronto. His research areas are design and fabrication of M/NEMS devices and micro-nanorobotic manipulation of biomaterials and nanomaterials.

Dr. Sun is a recipient of University of Minnesota Dissertation Fellowship and the Ontario Early Researcher Award for research in "MEMS-assisted micro and nanomanipulation of biological cells and nanomaterials." 